Tuchman, Gaye (1980): Die Verbannung der Frau in die symbolische Nichtexistenz durch die Massenmedien. In: Fernsehen und Bildung, 14 (1 - 2), S. $10-43$.

Weiderer, Monika (1993): Das Frauen- und Männerbild im Deutschen Fernsehen. Eine inhaltsanalytische Untersuchung der Programme von ARD, ZDF und RTL plus. Regensburg: S. Roderer Verlag.

Werner, Petra; Rinsdorf, Lars (1998): Ausgeblendet? - Frauenbild und Frauenthemen im nordrhein-westfälischen Lokalfunk. Schriftenreihe Medienforschung der LfR 27. Opladen: Leske + Budrich.

\section{Jürgen Grimm}

\section{Fernsehgewalt: Zuwendungsattraktivität - Erregungsverläufe - sozialer Effekt}

Zur Begründung und praktischen Anwendung eines kognitiv-physiologischen Ansatzes der Medienrezeptionsforschung am Beispiel von Gewaltdarstellungen

Opladen/Wiesbaden: Westdeutscher, 1999. $812 \mathrm{~S}$.

\section{ISBN 3-531-12668-7}

Jürgen Grimm erhebt den Anspruch, die Auswirkungen von Fernsehgewalt auf die $\mathrm{Zu}-$ schauer umfassend $\mathrm{zu}$ untersuchen: "Gewaltdarstellungen im Fernsehen [...] werden in dieser Studie aus der Zuschauerperspektive betrachtet" (S. 9). So formuliert der Autor zwar einen generellen Erklärungsanspruch, in den vorgestellten Untersuchungen werden jedoch nur die Wirkungen von Spielfilmgewalt, nicht jedoch von Darstellungen realer Gewalt (beispielsweise in Fernsehnachrichten) behandelt. (Allerdings verweist der Autor auf hierzu geplante Publikationen.) In drei „Experimenten" - da der Autor nicht unabhängige Variablen manipuliert, sondern lediglich Gruppen mit niedrigen und hohen Ausprägungen „psychosozialer" Merkmale miteinander vergleicht bzw. Korrelationen berechnet, handelt es sich lediglich um quasiexperimentelle Untersuchungen („Feldstudien“; vgl. Roth, 1993) wurden den Zuschauern vorzugsweise Szenen mit „gesteigerter“ Gewalt vorgegeben. Zwar ist die Wahrscheinlichkeit groß, dass solche Szenen von intensiven emotionalen Zustandsveränderungen bei den Rezipienten begleitet sind, andererseits muss man festhalten, dass solche Darstellungen im alltäglichen Fernsehprogramm eine Ausnahme darstellen oder gar nicht (mehr) vorkommen. (Nebenbei bemerkt: In meiner Sicht der Dinge wäre vielmehr eine Auseinandersetzung mit den weniger intensiven und eher als Stimmungsveränderungen denn als Emotionen zu beschreibenden Effekten von weniger spektakulären, aber weiter verbreiteten Gewaltdarbietungen wünschenswert. Allerdings stellt im Gegensatz zu starken emotionalen Medienwirkungen gerade die Erforschung von Emotionen im Niedrig-IntensitätsBereich ganz besondere Anforderungen an Theorie und Methodik.)

Grimm geht es um die Wirkungen der „Fernsehgewaltrezeption auf die Einstellungen der Zuschauer" (S. 9), dabei sind langfristige (überdauernde) Einstellungsveränderungen aufgrund der von ihm gewählten Versuchsanordnung gar nicht nachweisbar. In der Auswertung sucht Grimm nach systematischen Zusammenhängen zwischen der Trias $B e-$ schaffenheit des Filmmaterials ( $=$ Art der Gewalt), Rezeptionsvoraussetzungen (Nutzungsmotive, Persönlichkeitsdimensionen) und Wirkungen (Einstellungsveränderungen, physiologische Reaktionen). Damit steht der Ansatz des Autors auf einer Stufe mit dem „Uses-and-Effects"-Ansatz von Rubin (1994). Leider sehe ich bei der Vorgehensweise des Autors eine Reihe solch gravierender methodischer Mängel, dass ich auf eine inhaltliche Würdigung verzichten (vgl. die Zusammenfassung der Ergebnisse auf den Seiten $706 \mathrm{ff}$.) und mich im Folgenden auf ausgewählte Problempunkte konzentrieren möchte, die nach meiner Beurteilung die Generalisierbarkeit der Befunde in Frage stellen.

(i) Nutzungsmotive und individuelle Dispositionen werden mit Fragebögen erfasst, die Begleitumstände und kurzfristigen Nachwirkungen der Gewaltrezeption sowohl mit Fragebögen als auch durch physiologische Messungen beurteilt. Bei der Auswertung der Fragebogendaten hätte man sich vom Autor eine kritischere Einstellung zu Self-Report-Daten - wie sie beispielsweise von Vitouch (1997) vorgebracht wurden - gewünscht. $\mathrm{Da}$ in umfangreichem Maße Fragebogenergebnisse statistisch miteinander verglichen wurden (vgl. die Variablenaufstellung in Abschnitt 5.2.3), wäre ein Hinweis auf das Problem der statistischen Mehrfachtestung - bei einem a von 0,05 überschrei- 
ten immerhin 5 von 100 Tests allein per Zufall die Signifikanzgrenze - angebracht gewesen.

(ii) Im Rahmen des sog. „kognitiv-physiologischen Forschungsansatzes" interpretiert Grimm gleich- bzw. gegensinnige Verläufe der Werte von Hautleitfähigkeit (SCL) und Herzfrequenz (HF), indem er im Anschluss an Gray (1982) und Fowles (1980) zwischen der Aktivierung des Organismus durch das Behavioral Activation System (BAS) - angezeigt durch eine ansteigende Herzfrequenz - und der Erregung durch das Behavioral Inhibition System (BIS), die mit einer ansteigenden Hautleitfähigkeit einhergeht, unterscheidet. Während eine in der Regel durch Angstreize angeregte BIS-Aktivität kognitive Tätigkeit auslöst, führt eine verstärkte BAS-Aktivität zu Flucht- und Kampfreaktionen (oder zu belohnungsorientierter Annäherung). Das Problem bei einer solchen Vorgehensweise - SCL-Anstiege werden mit Kognition und Angst, HF-Anstiege mit Kampf und Flucht assoziiert - liegt darin, dass die Validität der Dateninterpretation vollständig von der Gültigkeit des Ansatzes von Gray und Fowles abhängig ist. Jedoch hat dieser als Brückentheorie dienende Ansatz nach meiner Kenntnis bislang (noch?) keine hinreichende Bestätigung gefunden und liegt vermutlich deshalb psychophysiologischen Forschungsarbeiten eher selten zugrunde. Insofern würde ich die Schlussfolgerungen aus den physiologischen Datenverläufen mit Vorsicht behandeln.

(iii) In der psychophysiologischen Forschung wird ausführlich die Problematik der Extraktion geeigneter Parameter (z. B. Maße der Variabilität der HF - die Standardabweichung ist hier als Indikator weniger geeignet - oder Hautleitfähigkeitsreaktionen SCR) diskutiert. Die vom Autor gegebene Interpretation von Robwertverläufen geht deutlich hinter die Ergebnisse dieser Diskussion zurück. Probleme sind auch mit der intraindividuellen Standardisierung (z. B. mit der Wahl eines geeigneten Referenzwertes) verbunden. Von wirklich exotischer Qualität sind in meinen Augen jedoch die Berechnungen des Autors, in denen er Werte der Hautleitfähigkeitsvariablen mathematisch zu Werten der Herzfrequenzvariablen in Beziehung setzt. (Mir drängt sich dabei die Assoziation zwischen Äpfeln und Birnen auf ...)

(iv) Ein letzter hier erörterter Kritikpunkt betrifft die zufallskritische Absicherung der Inter- pretation der physiologischen Verlaufskurven im Hinblick auf Gleich- oder Gegensinnigkeit. Die Interpretation von Verlaufskurven stellt ein intensiv diskutiertes und schwieriges Unternehmen dar (vgl. z. B. Watt, 1994; Mangold, Winterhoff-Spurk, Hamann \& Stoll, 1998). Unbeeindruckt von solchen statistischen Problemaspekten interpretiert Grimm die von ihm gemessenen Kurven nach seinem Eindruck und verlässt damit den Pfad der intersubjektiven Vergleichbarkeit. Wie kann der Autor begründen, welche in den Verlaufsdiagrammen gefundenen auf- bzw. absteigenden Kurvenabschnitte statistisch bedeutsam sind und welche lediglich Zufallsprodukte darstellen?

Das Erscheinen der Arbeit von Grimm habe ich seit langer Zeit mit Spannung erwartet. (Nachdem das Buch vom Verlag Mitte 1997 angekündigt worden war, habe ich es im Dezember 1997 bestellt, im Frühjahr 1999 jedoch wegen der langen Wartezeit wieder abbestellt. Im Sommer 1999 ist es dann erschienen und liegt mir jetzt vor. Vielleicht sollte in diesem Fall der Verlag seine Vertriebspolitik einmal überdenken.) Die Anschaffung des immerhin 98 DM teueren und die (zeitaufwendige) Lektüre des 812 Seiten starken und eng bedruckten Werkes empfehle ich nicht. Es überrascht mich, dass andere Autoren den Grimm'schen Ansatz loben; so schreibt Merten (1999) zu einer früheren (vergleichbaren) Studie des Autors: „Das von Grimm (1993) realisierte Experiment ist methodisch sehr sorgfältig durchgeführt worden. Insbesondere die physiologische Messung emotionaler Befindlichkeit ist hier hervorzuheben“ (S. 151). Kann diese diskrepante Einschätzung darauf zurückgehen, dass hier unterschiedliche methodische Auffassungen zweier mit Medienforschung befasster wissenschaftlicher Disziplinen aufeinander treffen? Ist die Situation dadurch charakterisiert, dass sich ein Medienwissenschaftler auf das (schwierige) Terrain der psychophysiologischen Erforschung von Medienwirkungen begibt und dafür Lob in seinem Fach erhält, während Kolleginnen und Kollegen aus der (Psycho-)Physiologie über das mangelnde Problembewusstsein und die Naivität nur den Kopf schütteln können, mit der physiologische Datenverläufe ausgewertet und interpretiert werden? Wohlgemerkt: Bei dieser (zugegebenermaßen) überspitzten Charakterisierung der Situation geht es mir auf keinen Fall darum, einem Medienwissenschaftler untersagen zu wollen, sich auch 
(medien-) psychophysiologisch zu betätigen. Jedoch erwarte ich in einem solchen Fall, dass er sich mit der gesamten Problematik auseinander setzt und sich nicht nur die Rosinen herauspickt.

Sollte ich mit der vorliegenden Buchbesprechung tatsächlich auf ein Problem im Miteinander von Medienwissenschaft und Medienpsychologie gestoßen sein, dann wäre anzuraten, dass jede Disziplin ihre eigenen methodischen Standards definiert und interdisziplinär diskutiert. Erste Schritte in dieser Richtung scheinen mir mit der Gründung der Fachgruppe „Methoden der Publizistik und Kommunikationsforschung " in der DGPuK und der Fachgruppe „Medienpsychologie“ in der Deutschen Gesellschaft für Psychologie getan zu sein. Sollte ein solcher Kommunikationsprozess weiter in Gang kommen, dann hätte auch das Buch von Grimm seinen guten $Z$ weck erfüllt.

Roland Mangold

\section{Literatur:}

Fowles, D. C. (1980). The three arousal model: Implications of Gray's two-factor-learning theory for heart rate, electrodermal activity, and psychopathy. Psychophysiology, 17, $87-104$.

Gray, J. A. (1982). The neuropsychology of anxiety: An inquiry into the functions of the septohippocampal system. Oxford: Clarendon Press.

Grimm, J. (1993). Der kultivierte Schrecken? Erlebnisweise von Horrorfilmen im Rahmen eines Zuschauerexperiments. Publizistik, 38, $206-216$.

Mangold, R., Winterhoff-Spurk, P., Hamann, G. \& Stoll, M. (1998). Veränderungen des zerebralen Blutflusses bei der Rezeption emotionalisierender Filmausschnitte: Eine Pilotstudie. Medienpsychologie, 10, 51 - 72.

Merten, K. (1999). Gewalt durch Gewalt im Fernsehen? Opladen: Westdeutscher Verlag.

Roth, E. (1993). Sozialwissenschaftliche Methoden. Lebr- und Handbuch für Forschung und Praxis. München: Oldenbourg.

Rubin, A. M. (1994). Media uses and effects: A uses-and-gratifications perspective. In J. Bryant \& D. Zillmann (eds.), Media effects: Advances in theory and research (pp. 447 - 436). Hillsdale: Erlbaum.
Tinchon, H.-J. (1999). Ein psychophysiologischer Meßplatz zur Unterstützung medienpsychologischer Fragestellungen. $\mathrm{Me}$ dienpsychologie, 11, 94.

Vitouch, P. (1997). Psychophysiological methods in media research. In P. WinterhoffSpurk \& T. H. A. van der Voort (Eds.), New horizons in media psychology. Research cooperation and projects in Europe (pp. 116-125). Opladen: Westdeutscher Verlag.

Watt, J. H. (1994). Detection and modeling of time-sequenced processes. In A. Lang (Ed.), Measuring psychological responses to media (pp. 181 - 207). Hillsdale: Erlbaum.

\section{Brit Großmann}

\section{Medienrezeption}

Bestehende Ansätze und eine konstruktivistische Alternative

Opladen/Wiesbaden: Westdeutscher Verlag, 1999. - 262 S.

ISBN 3-531-13377-2

Die Klage über die fehlende theoretische Grundlegung der Medienforschung wurde und wird vielfach erhoben. Sie bildet auch den Anknüpfungspunkt für die Überlegungen, die im vorliegenden Band angestellt werden. Hier wird allerdings nicht lediglich ein Klagelied angestimmt, um dann zur Tagesordnung überzugehen und die kritisierte Theorielosigkeit kommunikationswissenschaftlicher Forschungen weiter zu verwalten. Vielmehr sucht die Autorin einen Ausweg aus der Misere, und dies in einem Bereich, in dem ein besonderer Bedarf an einer aufarbeitenden Übersicht und Systematisierung besteht: im Bereich der Medienrezeptionsforschung. Nach einer knappen Einleitung im ersten Kapitel entwickelt Großmann in Kapitel 2 ein Evaluationsraster, mit dem relevante Forschungsansätze individueller Rezeption massenmedialer Kommunikationsangebote ausgewählt und bearbeitet werden. Ausgewählt werden Forschungen individueller Rezeptionsprozesse von Massenkommunikation, die umfassende theoretische Konzepte vorlegen und dabei individuelle und soziokulturelle Variablen sowie Merkmale der Massenkommunikation berücksichtigen und sich zugleich auf die Dynamik von Rezeptionsprozessen richten (16). In den Maschen des ausgelegten Netzes verfangen sich schließlich: das dynamisch-transaktionale Modell (Schönbach und 